\title{
ON WEAK SOLUTIONS OF RANDOM DIFFERENTIAL INCLUSIONS
}

\author{
MARIUSZ MICHTA ${ }^{1}$ \\ Technical University \\ Institute of Mathematics \\ Podgorna 50, 65-246 Zielona Gora \\ Poland
}

(Received January, 1995; Revised April, 1995)

\begin{abstract}
In the paper we study the existence of solutions of the random differential inclusion

$$
\begin{aligned}
& \dot{x}_{t} \in G\left(t, x_{t}\right) \quad P .1, t \in[0, T] \text {-a.e. } \\
& x_{0} \stackrel{d}{=} \mu
\end{aligned}
$$

where $G$ is a given set-valued mapping value in the space $K^{n}$ of all nonempty, compact and convex subsets of the space $\mathbb{R}^{n}$, and $\mu$ is some probability measure on the Borel $\sigma$-algebra in $\mathbb{R}^{n}$. Under certain restrictions imposed on $F$ and $\mu$, we obtain weak solutions of problem $(I)$, where the initial condition requires that the solution of $(I)$ has a given distribution at time $t=0$.
\end{abstract}

Key words: Set-Valued Mappings, Hukuchara's Derivative, Aumann's Integral, Tightness and Weak Convergence of Probability Measures.

AMS (MOS) subject classifications: 93E03, 54C65, 60B10.

\section{Preliminaries}

Problems of existence of solutions of differential inclusions were studied by many. In particular, random cases were considered in [3], [5], [7]. This work deals with the inclusion with a purely stochastic initial condition. First, we recall several notions and results needed in the sequel. Let $K_{c}(S)$ be the space of all nonempty compact and convex subsets of a metric space $S$ equipped with the Hausdorff metric $H$ (see e.g., [1], [4]): $H(A, B)=\max (\bar{H}(A, B), \bar{H}(B, A)) ; A, B \in K_{c}(S)$, where $\bar{H}(A, B)=\sup _{a \in A} \inf _{b \in B} \rho(a, b)$. By $\|A\|$ we denote the distance $H(A, 0)$. For $S$ being a separable Banach space, $\left(K_{c}(S), H\right)$ is a polish metric space.

Let $I=[0, T], T>0$. For a given multifunction $G: I \rightarrow K_{c}(S)$ by $D_{H} G\left(t_{0}\right)$, we denote its Hukuchara derivative at the point $t_{0} \in I$ (see e.g., [2], [9]) by the limits (if they exist in $K_{c}(S)$ )

$$
\lim _{h \rightarrow 0^{+}} \frac{F\left(t_{0}+h\right)-F\left(t_{0}\right)}{h}, \lim _{h \rightarrow 0^{+}} \frac{F\left(t_{0}\right)-F(t-h)}{h},
$$

both equal to the same set $D_{H} F\left(t_{0}\right) \in K_{c}(S)$.

\footnotetext{
${ }^{1}$ This work was supported by KBN grant No. 332069203.
} 
For $S=\mathbb{R}^{n}$ and $K^{n}=K_{c}\left(\mathbb{R}^{n}\right)$, we denote by $C_{I}=C\left(I, K^{n}\right)$ the space of all $H$-continuous setvalued mappings. In $C_{I}$ we consider a metric $\rho$ of uniform convergence

$$
\rho(F, G):=\sup _{0 \leq t \leq T} H(X(t), Y(t)), \text { for } X, Y \in C_{T} .
$$

Then $C_{I}$ is a polish metric space.

Let $(\Omega, F, P)$ be a given complete probability space. We recall now the notion of a multivalued stochastic process. The family of set-valued mappings $X=\left(X_{t}\right)_{t \geq 0}$ is said to be a multivalued stochastic process if for every $t \geq 0$, the mapping $X_{t}: \Omega \rightarrow K^{n}$ is measurable, i.e., $\bar{X}_{t}(U):=\{\omega$ : $\left.X_{t}(\omega) \cap U \neq \emptyset\right\} \in \mathcal{F}$, for every open set $U \subseteq \mathbb{R}^{n}$ (see e.g., $[1,4]$ ). It can be noted that $U$ can be also chosen as closed or Borel subset. We restrict our interest to the case when $0 \leq t \leq T, T>0$. If the mapping $t \rightarrow X_{t}(\omega)$ is continuous ( $H$-continuous) with probability on $(P .1)$, then we say that the process $X$ has continuous "paths."

Let us notice that the set-valued stochastic process $X$ can be though as a random element $X$ : $\Omega \rightarrow C_{I}$. Indeed, it follows immediately from [3] and from the fact that the topology of the uniform convergence and the compact-open topology in $C_{I}$ are the same.

Definition 1: A probability measure $\mu$ (on $C_{I}$ ) is a distribution of the set-valued process $X=\left(X_{t}\right)_{0 \leq t \leq T}$ if one has $\mu(A)=P(\bar{X}(A))$ for every Borel subset $A$ from $C_{I}$.

A distribution of $X$ will be denoted by $P^{X}$.

Definition 2: A set-valued mapping $F: I \times K^{n} \rightarrow K^{n}$ is said to be an integrably bounded of the

Caratheodory type if:
1) there exists a measurable function $m: I \rightarrow \mathbb{R}_{+}$such that $\int_{0}^{T} m(t) d t<\infty$ and
$\|F(t, A)\| \leq m(t) t$-a.e., $A \in K^{n}$.

2) $\quad F(t, \cdot)$ is $H$-continuous $t$-a.e.

3) $\quad F(\cdot, A)$ is a measurable multifunction for every $A \in K^{n}$.

Let us consider now the multivalued random differential equation:

$$
\begin{aligned}
& D_{H} X_{t}=F\left(t, X_{t}\right) P .1, t \in[0, T] \text {-a.e. } \\
& X_{0} \stackrel{d}{=} \mu
\end{aligned}
$$

where the initial condition requires that the set-valued solution process $X=\left(X_{t}\right)_{t \in I}$ has a given distribution $\mu$ at the time $t=0$. By a weak solution of $(I I)$ we understand a system $\left(\Omega, \mathcal{F}, P\left(X_{t}\right)_{t \in I}\right)$ where $\left(X_{t}\right)_{t \in I}$ is a set-valued process on some probability space $(\Omega, \mathcal{F}, P)$ such that $(I I)$ is met.

We state the following theorem (see e.g. [6]).

Theorem 1: Let $F: I \times K^{n} \rightarrow K^{n}$ be an integrably bounded set-valued function of the Caratheodory type and let $\mu$ be an arbitrary probability measure on the space $K^{n}$. Then there exists a weak solution of $(I I)$.

\section{Weak Solutions of Random Differential Inclusions}

As an application of Theorem 1, we show the existence of a weak solution of the random differential inclusion

$$
\begin{aligned}
& \dot{x}_{t}=G\left(t, x_{t}\right) \quad P .1, t \in[0, T] \text {-a.e. } \\
& x_{0} \stackrel{d}{=} \mu .
\end{aligned}
$$


The weak solution of $(I)$ is understood similarly as above, where $\mu$ is now a given probability measure on $\mathbb{R}^{n}$.

Let $\mathcal{\sigma}_{0}$ denote the family of nonempty open subsets of $\mathbb{R}^{n}$, and let $C=\left\{C_{V} ; V \in \mathcal{T}_{0}\right\}$, where $C_{V}=\left\{K \in K^{n}: K \cap V \neq \emptyset\right\}$. Then we have that $\mathscr{B}^{n}=\sigma(C)$ (see e.g. Proposition 3.1 [4]), where $\mathscr{B}^{n}$ is a Borel $\sigma$-field induced by the metric space $\left(K^{n}, H\right)$.

Lemma 1: The following hold true:

i) $K^{n} \in C$,

ii) if $A_{1}, A_{2}, \ldots \in C$ then $\bigcup_{n=1}^{\infty} A_{n} \in C$,

iii) if $C_{V_{1}} \subseteq C_{V_{2}} \subseteq \ldots$ then $V_{1} \subseteq V_{2} \subseteq \ldots$

Proof: The property $i$ ) is obvious. Let $V_{1}, V_{2}, \ldots, \in \mathcal{T}_{0}$ be such that $A_{n}=C_{V_{n}}$ for $n=1$, $2, \ldots$. To establish $i i$ ), let us observe that $\bigcup_{n=1}^{\infty} A_{n}=C \bigcup_{n=1}^{\infty} V_{n}$.

Let us suppose that iii) does not hold. Then for some $k \geq 1, V_{k} \nsubseteq V_{k+1}$. Hence there exists a point $x \in V_{k}$ such that $x \notin V_{k+1}$. But then $\{x\} \in C_{V_{k}}$ and $\{x\} \notin C_{V_{k+1}}$ contradicts to $C_{V_{k}} \subseteq C_{V_{k+1}}$

To obtain our main result we need the following lemma:

Lemma 2: If $\mu$ is a probability measure on the Borel $\sigma$-algebra $\mathfrak{B}\left(\mathbb{R}^{n}\right)$, then there exists a probability measure $\widehat{\mu}$ on the space $K^{n}$ such that $\widehat{\mu}\left(C_{V}\right)=\mu(V), V \in \mathcal{T}_{0}$.

Proof: Let $C$ be the family generating Borel $\sigma$-field $\mathfrak{B}^{n}$. We define a set-function $\nu$ on $C$ by $\nu\left(C_{V}\right)=\mu(V)$. Let us observe that $\nu$ is well-defined. Indeed, if $C_{V_{1}}=C_{V_{2}}$ and $\mu\left(V_{1}\right) \neq \mu\left(V_{2}\right)$ then $V_{1} \neq V_{2}$. Hence $V_{1} \backslash V_{2} \neq \emptyset$ or $V_{2} \backslash V_{1} \neq \emptyset$. Without loss of generality we may assume the first case. Then there exists $x \in V_{2}$ such that $x \notin V_{1}$. But then $\{x\} \in C_{V_{2}}$ and $\{x\} \notin C_{V_{1}}$ which contradicts with an equality $C_{V_{1}}=C_{V_{2}}$. Similarly, it can be shown that if the sets $C_{V_{1}}$ and $C_{V_{2}}$ are disjoint, then the sets $V_{1}, V_{2}$ have the same property too. Hence we get $\nu\left(C_{V_{1}} \cup\right.$ $\left.C_{V_{2}}\right)=\nu\left(C_{V_{1}}\right)+\nu\left(C_{V_{2}}\right)$ for disjoint $C_{V_{1}}$ and $C_{V_{2}}$. From Lemma 1 we conclude that, if $C_{V_{1}} \subseteq$ $C_{V_{2}} \subseteq \ldots$, then

$$
\bigcup_{n=1}^{\infty} C_{V_{n}} \in C \text { and } \nu\left(\bigcup_{n=1}^{\infty} C_{V_{n}}\right)=\lim \nu\left(C_{V_{n}}\right)
$$

Moreover, $\nu\left(K^{n}\right)=1$. Finally let us observe that $\nu$ is $\sigma$-subadditive. Next we define another set function $\widehat{\nu}$ as follows:

$$
\widehat{\nu}(A):=\inf \{\nu(D): A \subset D, D \in C\}, A \subset K^{n} .
$$

Standard calculations show that $\widehat{\nu}$ is an outer measure on $K^{n}$. Thus from the Caratheodory Theorem, $\widehat{\nu}$ is a probability measure on the $\sigma$-field of $\widehat{\nu}$-measurable subsets in $K^{n}$. Setting $\widehat{\mu}=$ $\left.\widehat{\nu}\right|_{\mathscr{B}^{n}}$, we obtain a desired probability measure.

We now present the following existence theorem.

Theorem 2: Let us suppose that $G: I \times \mathbb{R}^{n} \rightarrow K^{n}$ is an integrably bounded multifunction of the Caratheodory type. Then for any probability measure $\mu$ on $\mathbb{R}^{n}$, there exists a weak solution of problem $(I)$.

Proof: Lemma 2 yields the existence of a probability measure $\widehat{\mu}$ on the metric space $\left(K^{n}, H\right)$ with the property: $\widehat{\mu}\left(C_{V}\right)=\mu(V), V \in \mathcal{T}_{0}$. Let $F: I \times K^{n} \rightarrow K^{n}$ be a multifunction defined by $F(t, A)=\overline{c o} G(t, A)$, for $A \in K^{n}$. Hence from Lemma 1.1 [9], the set-valued mapping $F$ is integrably bounded of the Caratheodory type too. Consequently, by Theorem 1 , there exists a probability space $(\Omega, F, P)$ and the set-valued stochastic process $X=\left(X_{t}\right)_{0} \leq t \leq T$ (on it) with 
continuous "paths" and with values in $K^{n}$ which is a weak solution of the equation

$$
\begin{aligned}
& D_{H} X_{t}=F\left(t, X_{t}\right) \quad P .1, t \in[0, T] \text {-a.e. } \\
& X_{0} \stackrel{d}{=} \widehat{\mu} .
\end{aligned}
$$

From Kuratowski and Ryll-Nardzewski Selection Theorem [4] we can choose $\xi: \Omega \rightarrow \mathbb{R}^{n}$ as a measurable selection of $X_{0}$. Then by Theorem 4 [5] (see also [3]), there exists a stochastic process $x=\left(x_{t}\right)_{0 \leq t \leq T}$ as a selection of $X$ that is a solution (in strong sense) of the random differential inclusion:

$$
\begin{aligned}
& \dot{x}_{t} \in G\left(t, x_{t}\right) \quad P .1, t \in[0, T]-\text { a.e. } \\
& x_{0} \in U P .1
\end{aligned}
$$

where $U(\omega)=\{\xi(\omega)\}$ for $\omega \in \Omega$.

To complete the proof, it is sufficient to show that $x_{0} \stackrel{d}{=} \mu_{d}$ Let us notice that $\left\{\omega: x_{0}(\omega) \in\right.$ $V\}=\{\omega: \xi(\omega) \in V\} \subset\left\{\omega: X_{0} \cap V \neq \emptyset\right\}, V \in \mathcal{T}_{0}$. Because of $X_{0} \stackrel{d}{=} \widehat{\mu}$ and $\widehat{\mu}\left(C_{V}\right)=\mu(V)$ we have

$$
P^{x} 0(V) \leq \mu(V)
$$

Using regularity properties of probability measures (on a separable metric space) (see e.g., Th. 1.2 [8]), we have that

$$
P^{x} 0(B)=\inf \left\{P^{x} 0(V): B \subset V, V \in \mathcal{J}_{0}\right\}
$$

and $\mu(B)=\inf \left\{\mu(V): B \subset V, V \in \mathcal{T}_{0}\right\}$ for every Borel subset $B$ of $\mathbb{R}^{n}$. Hence from inequality (*) we get $P^{x_{0}}(B) \leq \mu(B)$. But $P^{x_{0}}$ and $\mu$ are probability measures. Therefore they have to be equal.

\section{References}

[1] Himmelberg, C.J. and Van Vleck, F.S., The Hausdorff metric and measurable selections, Topol. and its Appl. 20 (1985), 121-133.

[2] Hukuchara, M., Sur l application semicontinue dont la valeur est un compact convexe, Funkcial. Ekwac. 10 (1967), 43-66.

[3] Kandilakis, D.A. and Papageorgiou, N.S., On the existence of solutions of random differential inclusions in Banach spaces, J. Math. Anal. Appl. 126 (1987), 11-23.

[4] Kisielewicz, M., Differential Inclusions and Optimal Control, Kluwer 1991.

[5] Michta, M., Set-valued random differential equations in Banach space, Discussines Math. (1994), (submitted).

[6] Michta, M., Weak solutions of set-valued random differential equations, Demonstratio Mathematica (1994), (submitted).

[7] Nowak, A., Random differential inclusions: measurable selection approach, Ann. Pol. Math. XLIX (1989), 291-296.

[8] Parthasarathy, K.R., Probability Measures on Metric Spaces, Academic Press, New York 1967.

[9] Tolstonogov, A., Differential Inclusions in Banach Spaces, Nauka, Moscow 1986 (Russian). 


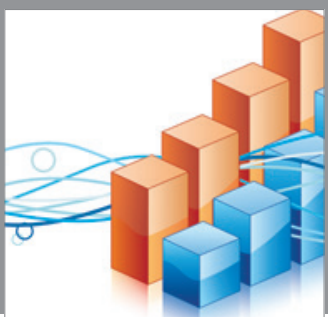

Advances in

Operations Research

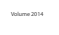

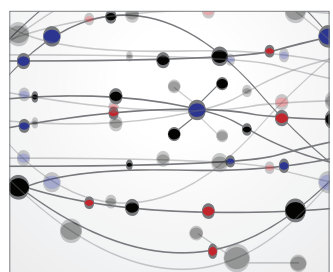

\section{The Scientific} World Journal
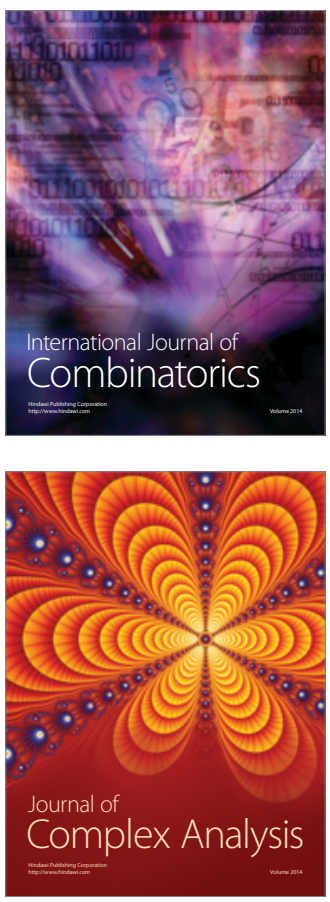

International Journal of

Mathematics and

Mathematical

Sciences
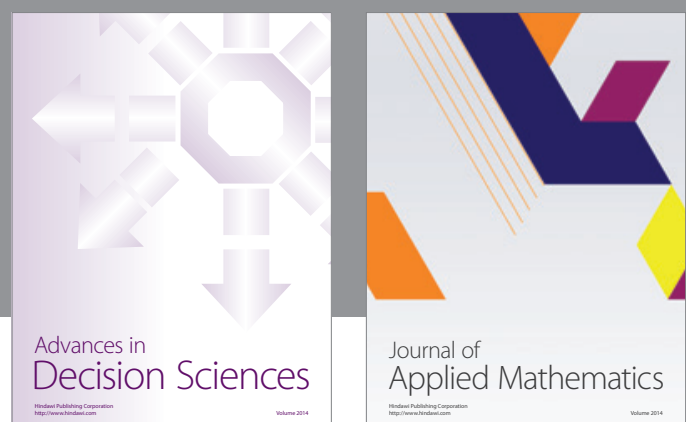

Journal of

Applied Mathematics
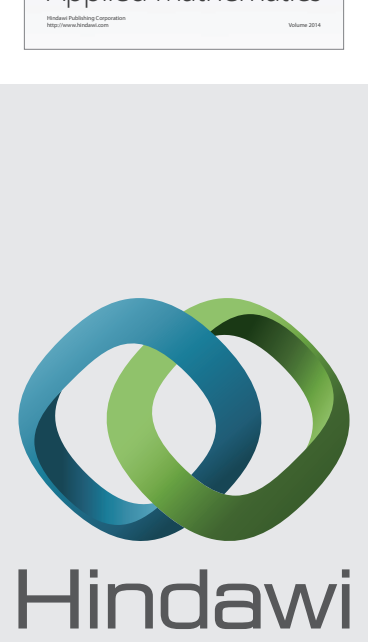

Submit your manuscripts at http://www.hindawi.com
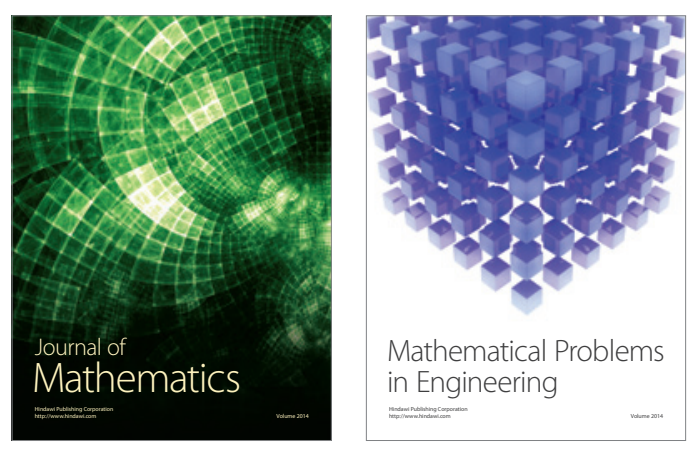

Mathematical Problems in Engineering
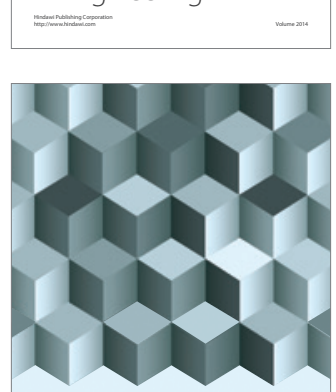

Journal of

Function Spaces
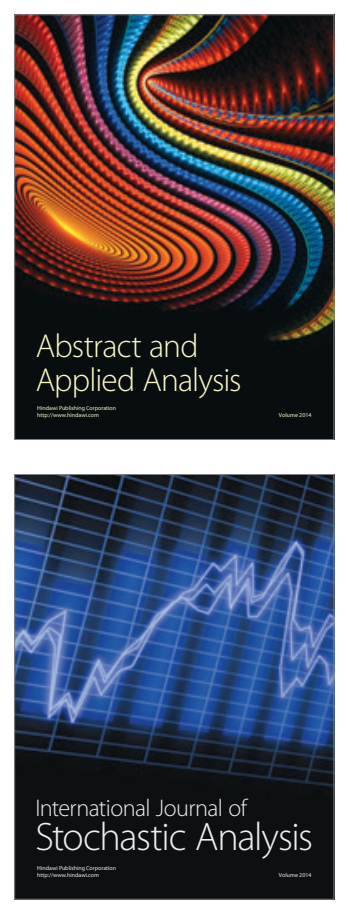

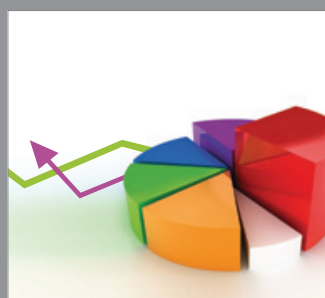

ournal of

Probability and Statistics

Promensencen
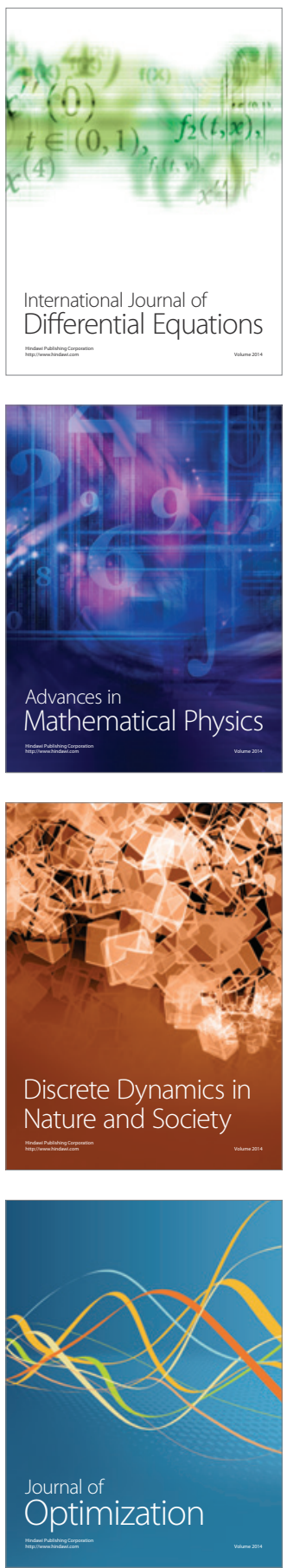